\title{
Denosumab Rapidly Increases Cortical Bone in Key Locations of the Femur: A 3D Bone Mapping Study in Women With Osteoporosis
}

\author{
Kenneth ES Poole, ${ }^{1}$ Graham M Treece, ${ }^{2}$ Andrew H Gee, ${ }^{2}$ Jacques P Brown, ${ }^{3}$ Michael R McClung, ${ }^{4}$ \\ Andrea Wang, ${ }^{4}$ and Cesar Libanati ${ }^{5}$ \\ ${ }^{1}$ Department of Medicine, University of Cambridge, Cambridge, UK \\ ${ }^{2}$ Department of Engineering, University of Cambridge, Cambridge, UK \\ ${ }^{3} \mathrm{CHU}$ de Québec Research Centre, Laval University, Quebec City, Canada \\ ${ }^{4}$ Oregon Osteoporosis Center, Portland, OR, USA \\ ${ }^{5}$ Amgen Inc., Thousand Oaks, CA, USA
}

\begin{abstract}
Women with osteoporosis treated for 36 months with twice-yearly injections of denosumab sustained fewer hip fractures compared with placebo. Treatment might improve femoral bone at locations where fractures typically occur. To test this hypothesis, we used 3D cortical bone mapping of postmenopausal women with osteoporosis to investigate the timing and precise location of denosumab versus placebo effects in the hips. We analyzed clinical computed tomography scans from 80 female participants in FREEDOM, a randomized trial, wherein half of the study participants received subcutaneous denosumab $60 \mathrm{mg}$ twice yearly and the others received placebo. Cortical 3D bone thickness maps of both hips were created from scans at baseline, 12, 24, and 36 months. Cortical mass surface density maps were also created for each visit. After registration of each bone to an average femur shape model followed by statistical parametric mapping, we visualized and quantified statistically significant treatment effects. The technique allowed us to pinpoint systematic differences between denosumab and control and to display the results on a 3D average femur model. Denosumab treatment led to an increase in femoral cortical mass surface density and thickness, already evident by the third injection (12 months). Overall, treatment with denosumab increased femoral cortical mass surface density by $5.4 \%$ over 3 years. One-third of the increase came from increasing cortical density, and two-thirds from increasing cortical thickness, relative to placebo. After 36 months, cortical mass surface density and thickness had increased by up to $12 \%$ at key locations such as the lateral femoral trochanter versus placebo. Most of the femoral cortex displayed a statistically significant relative difference by 36 months. Osteoporotic cortical bone responds rapidly to denosumab therapy, particularly in the hip trochanteric region. This mechanism may be involved in the robust decrease in hip fractures observed in denosumab-treated women at increased risk of fracture. $\odot 2014$ American Society for Bone and Mineral Research.
\end{abstract}

KEY WORDS: FEMORAL NECK FRACTURES; HIP FRACTURE; FEMALE; OSTEOPOROSIS; POSTMENOPAUSAL OSTEOPOROSIS; DENOSUMAB; COMPUTED TOMOGRAPHY; COMPUTATIONAL ANATOMY

\section{Introduction}

ombating the rising epidemic of hip fractures is a key goal in musculoskeletal research. The FREEDOM study was a large, randomized clinical trial comparing denosumab (the antireceptor activator of NF- $\kappa B$ ligand [RANKL] binding monoclonal antibody treatment) with placebo in postmenopausal women with osteoporosis. Women treated with denosumab injections sustained significantly fewer hip fractures than those receiving placebo. ${ }^{(1)}$ The difference in hip fracture events appeared early (Fig. 1), persisted, and was noteworthy in subjects aged 75 years and older. ${ }^{(1)}$ Most hip fractures in clinical practice occur because of falls onto the greater trochanter, ${ }^{(2)}$ and when femurs are subjected to similar conditions in the lab, the failure of very small (less than $6.4 \%$ of all bone tissue) foci of bone are sufficient to lead to catastrophic fracture. ${ }^{(3)}$ When FREEDOM study participants' femurs were subjected to sideways fall simulations, denosumab increased calculated femoral strength by $8.4 \%$ over 3 years, ${ }^{(4)}$ perhaps because of focal changes in bone structure. It is not yet clear how antiresorptives prevent hip fractures in the early phase of landmark clinical trials such as FREEDOM. It is possible that the dramatic effects in reducing hip fractures might be attributable to treatment effects on bone microstructure that are not detected with in vivo imaging techniques. We favor an

Received in original form August 20, 2014; revised form July 16, 2014; accepted July 30, 2014. Accepted manuscript online August 4, 2014.

Address correspondence to: Kenneth ES Poole, BM, FRCP, PhD, Department of Medicine, Box 157, Addenbrooke's Hospital, University of Cambridge, Cambridge, Cambridgeshire, UK CB2 0QQ. E-mail: kp254@nhs.net

Additional Supporting Information may be found in the online version of this article.

This is a Commentary on Roger Zebaze and Ego Seeman (J Bone Miner Res. 2015;30:24-29. DOI: 10.1002/jbmr.2419)

Journal of Bone and Mineral Research, Vol. 30, No. 1, January 2015, pp 46-54

DOI: $10.1002 / j b m r .2325$

(C) 2014 American Society for Bone and Mineral Research 

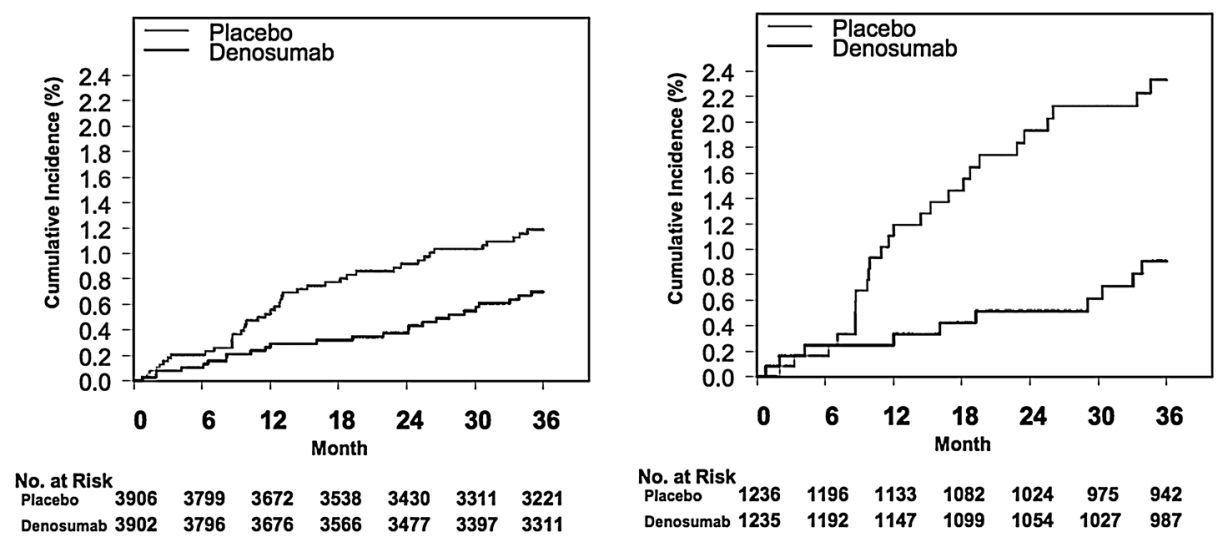

Fig. 1. Kaplan-Meier curves of time to first hip fracture in all women (left) and women aged 75 years and older (right) during the FREEDOM clinical trial.

alternative hypothesis, namely that beneficial effects of antiresorptive drugs can be detected in imaging data, particularly in focal areas known to be associated with hip fracture. To test this, we investigated the effects of denosumab treatment on cortical bone throughout the proximal femur, paying particular attention to the specific locations where hip fractures have been found to occur during laboratory simulations. ${ }^{(5-7)}$

Both cortical and trabecular bone compartments are critical in determining hip fracture resistance. ${ }^{(8-11)}$ We recently used 3D cortical bone mapping ${ }^{(12,13)}$ to analyze pelvic computed tomography (CT) scans from women with and without acute hip fracture. ${ }^{(14)}$ Women with a femoral neck fracture had a patch of focal cortical bone thinning in the superior head-neck junction of the femur, whereas women with a trochanteric hip fracture had a patch of focal cortical thinning in the lateral trochanteric region. ${ }^{(14)}$ Similar areas were also identified in a prospective study evaluating 3D density distributions in an Icelandic cohort who sustained hip fracture. ${ }^{(9)}$ Such defects are anatomically located in cortical and trabecular areas where femoral neck and trochanteric fractures occur during laboratory simulations, ${ }^{(15,16)}$ often coinciding with regions that receive low amounts of mechanical loading during habitual activities such as walking. ${ }^{(17)}$ Irrespective of their pathogenesis, focally thin cortical bone in the hip predicts hip fracture. ${ }^{(18)}$

In this study, we tested the hypothesis that denosumab would improve femoral cortical bone structure in those same areas known to be deficient and associated with increased hip fracture risk. To investigate this hypothesis, we used 3D cortical bone mapping ${ }^{(12)}$ to determine the precise location of denosumab effects in the femurs of women with osteoporosis, surmising that beneficial effects would correspond with the focally thin areas identified previously. ${ }^{(14)}$ We visualized the precise magnitude and time course of changes in the femoral cortex with denosumab therapy relative to placebo among participants in the FREEDOM clinical trial.

\section{Materials and Methods}

Cortical bone mapping ${ }^{(12)}$ (Fig. 2) allows us to display local, pointwise measurements of the thickness or mass surface density as a color map over a patient's 3D bone surface, with several thousand independent measurements across each proximal femur. Surface density is a regional measurement computed at each measurement point as $0.1 \times$ local cortical thickness $(\mathrm{mm})$ $\times$ average cortical bone mineral density $\left(B M D ; \mathrm{g} / \mathrm{cm}^{3}\right)$, and representing mass per unit surface area $\left(\mathrm{g} / \mathrm{cm}^{2}\right)$. When differences are expressed systematically between femurs from suitably sized groups of individuals, the technique has sufficient sensitivity to detect even small changes ( $\sim 30$ microns) in thickness with treatment ${ }^{(19)}$ or between cases and controls. ${ }^{(14)}$ We use it here to visualize the location of average denosumab treatment effects. From 2004 to 2009, women aged 60 to 90 years with osteoporosis defined by dual-energy X-ray absorptiometry (DXA) T-scores $\leq-2.5$ at the lumbar spine and/or proximal femur were consented and participated in the FREEDOM study, an international, randomized, placebo-controlled trial. Eligible women were randomly allocated to receive either $60 \mathrm{mg}$ denosumab or placebo by subcutaneous injection every 6 months for 3 years. All women received daily supplements of $1000 \mathrm{mg}$ calcium (minimum), and those with insufficient vitamin D were supplemented with between 400 and 800 IU vitamin D daily. Full details of the study population are available, ${ }^{(1)}$ but in brief, women currently taking oral or iv bisphosphonates were excluded, as were those taking strontium ranelate, parathyroid hormone, steroid hormone-replacement therapy (HRT), selective estrogen-receptor modulators (SERMs), calcitonin, or calcitriol. Participants at multiple qualified centers were invited to enroll in a QCT study and, after providing written informed consent, underwent standard clinical CT scanning at baseline, 12, 24, and 36 months. Participants were positioned on an image analysis phantom (Mindways calibration phantom, Mindways Software Inc., Austin, TX, USA), and a single CT scan including both hips was performed from $1 \mathrm{~cm}$ above the acetabulum to $2 \mathrm{~cm}$ below the lesser trochanter $(120 \mathrm{kV}$, pitch $=1,170 \mathrm{mAs}$, reconstruction field of view $400 \mathrm{~mm}$, medium kernel, reconstruction slice thickness $\leq 1.25 \mathrm{~mm}$ ). CT scan image quality control, scanner calibration, and stability were monitored by Synarc (Synarc, Hamburg, Germany). A full description of the imaging protocol and primary results has been published. ${ }^{(20)}$ After accounting for missing visits and image quality control, there were 80 hip scans at baseline, with 79 at 12 months, 78 at 24 months, and 62 at 36 months. Of 

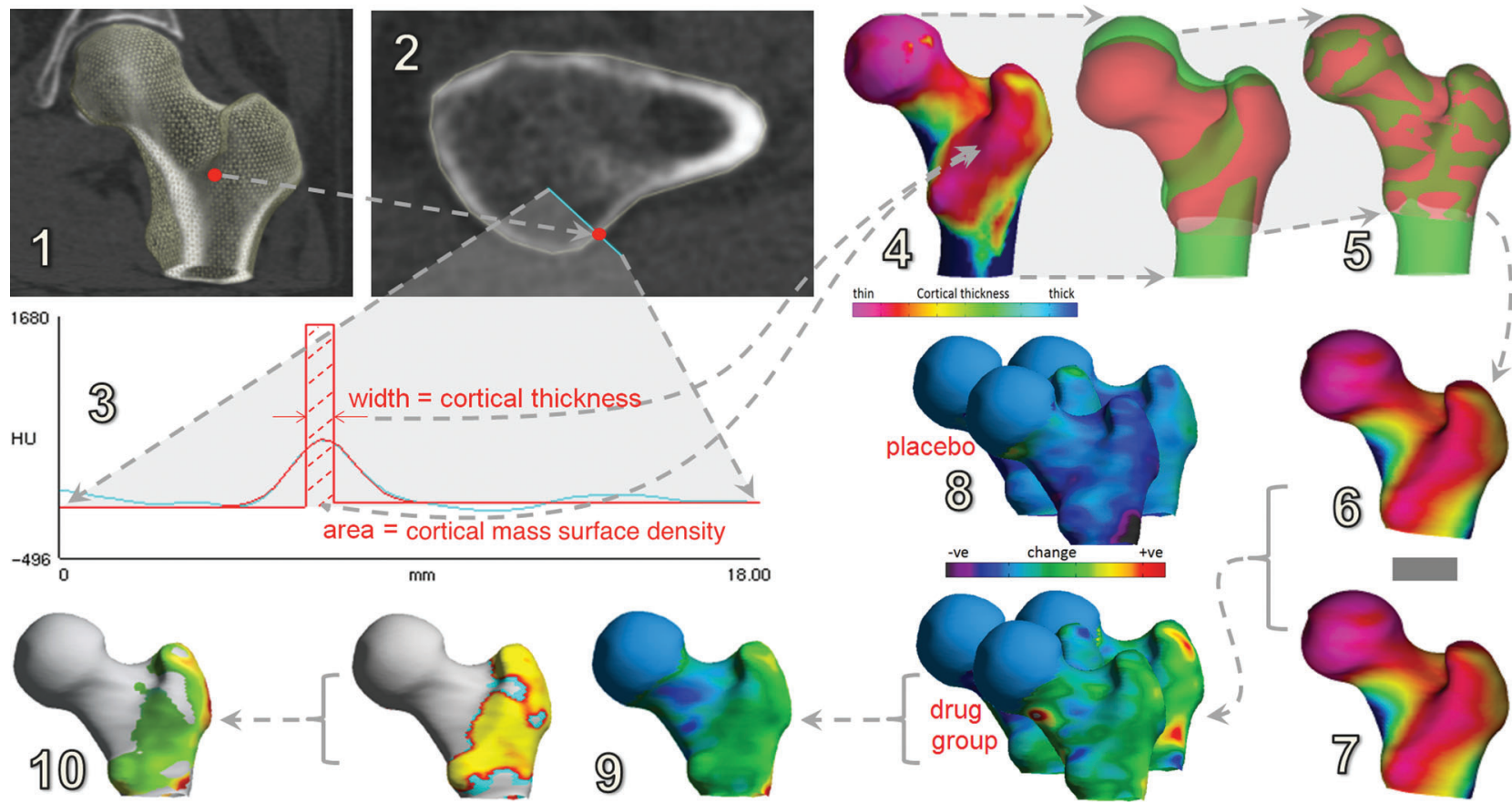

Fig. 2. Summary of the steps involved in $3 \mathrm{D}$ cortical bone mapping and analysis of changes over time using statistical parametric mapping (SPM). (1) Triangulated 3D surface of a left proximal femur (one individual) and corresponding coronal CT plane after segmentation with Stradwin software. (2) An axial CT slice to demonstrate sampling the CT data along an 18-mm cyan transcortical line at the surface location indicated by the red circular dot. (3) Parametric fit (red curve) and original CT data (cyan curve) superimposed on a graph of distance ( $x$ axis) versus Hounsfield units ( $y$ axis) along the 18-mm transcortical line. The cortical thickness is the distance between the red arrows. (4) Many thousands of cortical thickness estimates painted by reference to a color scale onto the femoral surface of the same individual. (5) Registration (morphing) of the individual's femoral surface shape to the canonical femur shape (CFS). (6) Resultant map displaying cortical thickness for the individual now on the CFS after left and right femurs are averaged to give a single map per participant at each visit. (7) Subtracting the baseline cortical thickness map from the 36-month map in an individual to create a difference map. (8) After unblinding, there are two groups: cortical thickness difference maps from the placebo (upper) and denosumab groups (lower). (9) Typical results from one arm of the study (in this case, the denosumab group) using the statistical parametric mapping process to find and quantify where the 36-month maps differ from baseline maps. The left image shows a yellow $p$ value map displayed on the CFS. The yellow foci are vertices where cortical thickness changed significantly between baseline and 36 months, ranging from gray (nonsignificant) via orange to increasingly bright yellow as the $p$ value decreases. The right image shows the amount of change as a color scale on the CFS. In this hypothetical case, we see increasing thickness with denosumab. (10) Final result showing the magnitude of increase in cortical thickness on the CFS with only areas of statistically significantly greater cortical thickness retained, the rest grayed out. For actual results, see Figs. 4 and 5.

these, 78 were common between baseline and 12 months, 77 between baseline and 24 months, and 61 between baseline and 36 months. Anonymized CT scans were transferred to Cambridge, and all image processing and analysis steps were performed by operators blind to treatment allocation. Only tomographic reconstructions with a large field of view completely including the calibration phantom were considered.

The process for 3D cortical bone mapping is summarized in Fig. 2. Both femurs were segmented semiautomatically in Stradwin software (Treece, Gee, Cambridge, UK) before mapping cortical thickness at approximately 6000 surface points per femur. Cortical thickness and mass surface density were estimated from the CT data using the method described by Treece and colleagues. ${ }^{(12,13)}$ By making reasonable assumptions about both the anatomy and the imaging blur, thickness can be measured to super-resolution accuracy across the entire proximal femur. The methodology has been validated against thickness measurements obtained from high-resolution micro-CT scans of cadaveric femurs. ${ }^{(12,13)}$ The process of measuring cortical thickness and mass at every vertex on a 3D bone surface was as follows:

\section{Segmentation}

Both femurs in each data set were segmented in multiple slices (at least one every $3 \mathrm{~mm}$ ) of the DICOM data, and the segmentations used to create a regular triangulated proximal femoral surface for each femur. The subsequent measurement points were located at the vertices of these triangles.

\section{Estimating whole femur average cortical density}

A single value of average cortical density $\left(\mathrm{mg} / \mathrm{cm}^{3}\right)$ was estimated for the whole femur, as described in Treece and colleagues, ${ }^{(13)}$ by reference to the calibration standards in the Mindways phantom, converting Hounsfield units into $\mathrm{mg} / \mathrm{cm}^{3}$ of bone. This average cortical density value was generated from both femurs in an individual.

\section{Estimating cortical mass surface density and thickness}

At each of the several thousand vertices per femur, the cortical bone CT data were sampled by automated placement of an 
18-mm line normal to the surface at that point. This line cut from outside the bone, through the periosteum, then cortical bone, and into the endosteal and trabecular compartment. A plot of Hounsfield units sampled from the CT data on the $y$ axis against distance along the line on the $x$ axis is shown in Fig. 2. At each vertex, a six-parameter model constrained by the peak cortical bone Hounsfield value within that femur was matched to the sampled CT data through optimization, giving cortical thickness (Fig. 2). Multiplying that cortical thickness value $(\mathrm{mm})$ by the whole femur's average cortical density $\left(\mathrm{mg} / \mathrm{cm}^{3}\right)$ gave a value of cortical bone mass per unit surface area for each vertex $(0.1 \times \mathrm{CTh} \times$ CBMD). This unit is correctly referred to as surface density (defined as "a quantity [such as mass or electricity] per unit area distributed over a surface," Merriam-Webster Dictionary, 2014), and here can be thought of as the total amount of cortical bone (in mg) contained within the calculated endosteal and periosteal cortical boundaries beneath a given area (in $\mathrm{cm}^{2}$ ) of bone surface For an individual femur, either thickness or mass surface density can be visualized on the femur surface as a map using a color scale. ${ }^{(13)}$ Binary files containing the $x, y$, and $z$ coordinates of each vertex, plus the cortical mass surface density or thickness at each vertex were exported for analysis in Surfstat. A typical 18-mm-long transcortical surface normal and the corresponding parametric fit model, as well as the sampled CT data constrained by the peak cortical bone Hounsfield value of $1680 \mathrm{HU}$ can be found in Fig. 2 .

\section{Registration}

To account for variations in inter-subject morphology, each map was spatially realigned with a canonical femur surface using a B-spline free-form deformation calculated by the iterative closest point registration algorithm. ${ }^{(21)}$ This process maps the nearest thickness values to the vertices on the canonical femur surface. The spatially normalized maps were then smoothed with a $10-\mathrm{mm}$ full-width-half-maximum filter. The femoral head was excluded because the presence of the acetabulum made measurements here less reliable. The distal end of the analysis plane was defined by the extent of the common (canonical) shape.

\section{Difference maps}

Left and right hip cortical bone maps were averaged for each visit, creating a single cortical femur map per participant at baseline, 12-, 24-, and 36-month visits. Each participant's map from the 12-month visit was subtracted from her own baseline bone map to give a difference map, a process repeated for the 24- and 36-month visits. This resulted in 78 difference maps (from 78 participants) at 12 months, 77 difference maps at 24 months, and 61 difference maps at 36 months.

\section{Statistical parametric mapping (SPM) for significance and magnitude of differences}

Information from the study coordinating center was supplied, indicating which patients were in the denosumab group and which in the placebo group. Having sorted the difference maps into those from the drug group and those from the placebo group, we used SPM to find and quantify where the cortical mass surface density and thickness differed between baseline and each visit. Surfstat (http://math.mccgill.ca/keith/surfstat/) 22,23$)$ was used to test whether the difference value at each vertex was significantly different from zero using a fixed-effects general linear model. $T$-statistics were calculated to test the significance of differences. No allowance was made in the analysis for patient age, height, or any other demographics because prior and concurrent work have established not only that there were no important differences between these parameters in the FREEDOM QCT study group participants but also that denosumab effects show no age dependency. ${ }^{(24)}$ Random field theory then furnished $p$ values, corrected for multiple comparisons to control the overall imagewise chance of false positives. These methods are described fully in two recent articles. ${ }^{(14,19)}$ In the results figures, the canonical femur shape is viewed from anterior and posterior aspects for each visit to show the change in mass surface density $\left(\mathrm{g} / \mathrm{cm}^{2}\right)$ or in thickness $(\mathrm{mm})$ relative to baseline. We present difference and significance simultaneously, by retaining only those differences that were statistically significant and graying out the remainder.

Measuring the thickness of the fairly thin cortex of the proximal femur is challenging in clinical CT data. We have previously demonstrated that our cortical thickness measurement technique is more accurate than the existing alternatives; ${ }^{(12,13)}$ however, it is important to note that there remains some ambiguity between measured cortical thickness increases owing to the filling in of intracortical pores, or owing to increases in actual overall cortical thickness. Once blurred by the imaging system, these two mechanisms can produce identical CT data.
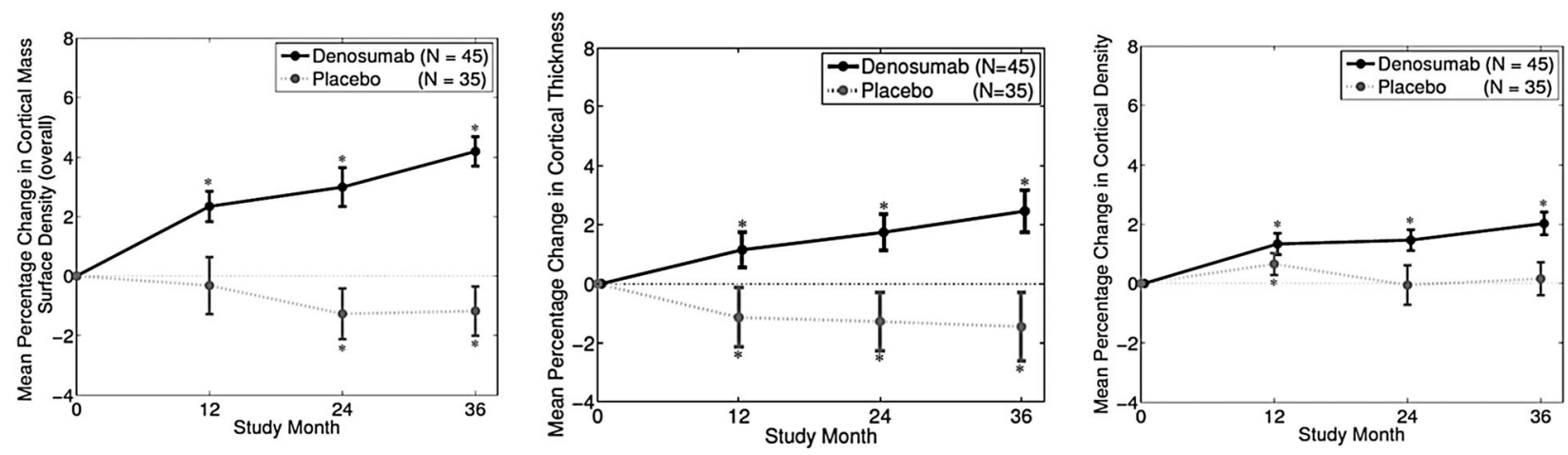

Fig. 3. Percentage changes in overall cortical mass surface density (left), overall cortical thickness (center), and overall cortical density (right) during 3 years' treatment with denosumab or placebo. These results are averaged across the entire femoral cortical surface. (By "overall," we denote the mean value for all femurs, each averaged over the entire canonical femur surface; ie, the average of approximately 6000 points per femur from 78 femurs [every femur]). The values are shown for each of four visits (baseline, 12 months, 24 months, and 36 months). 
Change in cortical mass $\left(\mathrm{mg} / \mathrm{cm}^{2}\right)$ surface density over 36 months

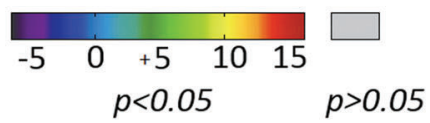

Percent change in cortical mass (\%) surface density over 36 months

$\begin{array}{rlrll}-5 & 0 & +5 & 10 & \\ & p<0.05 & & p>0.05\end{array}$

Change in cortical mass $\left(\mathrm{mg} / \mathrm{cm}^{2}\right)$ surface density over 36 months
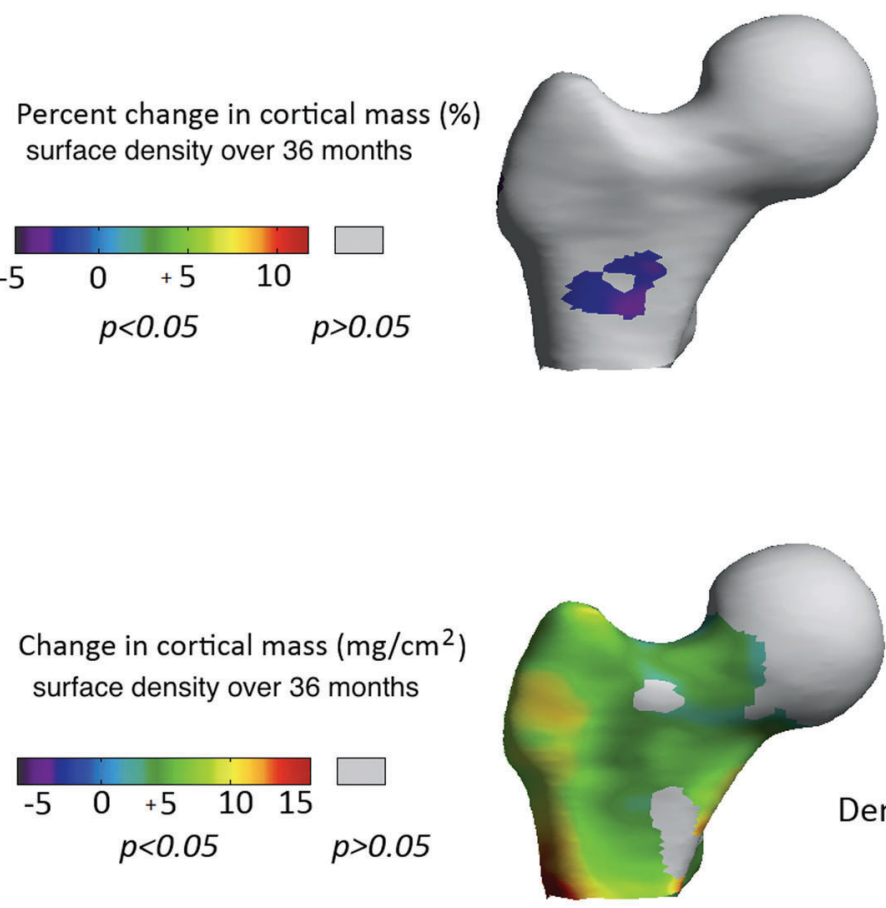

Placebo Group
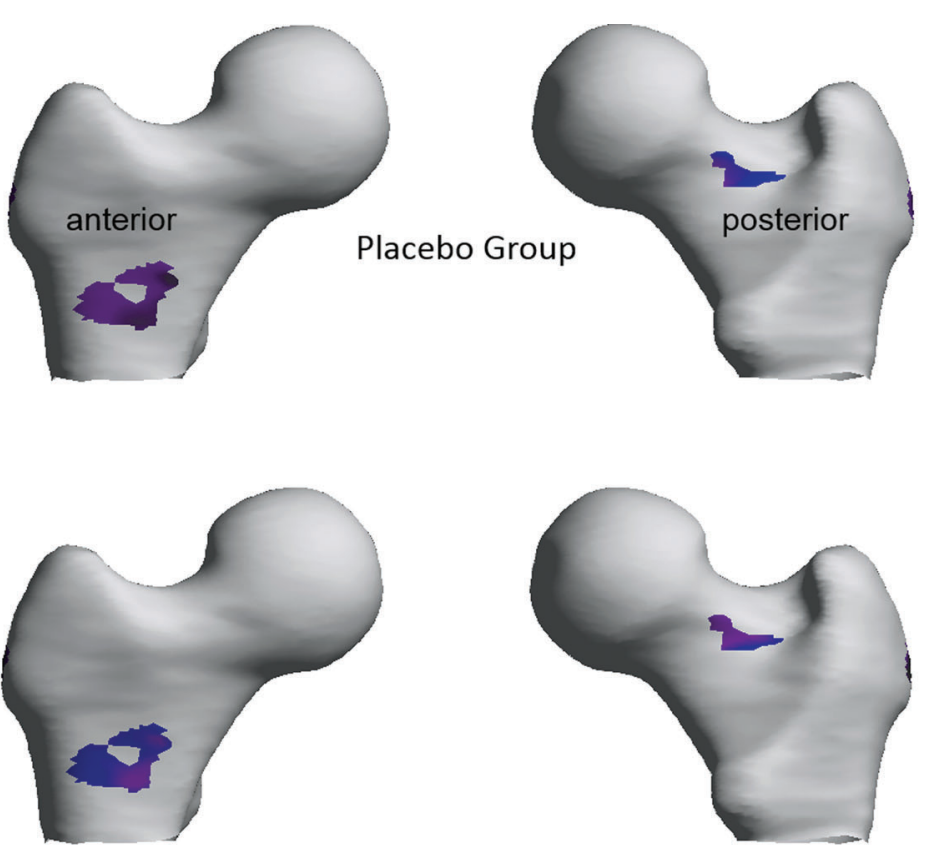

enosumab Group
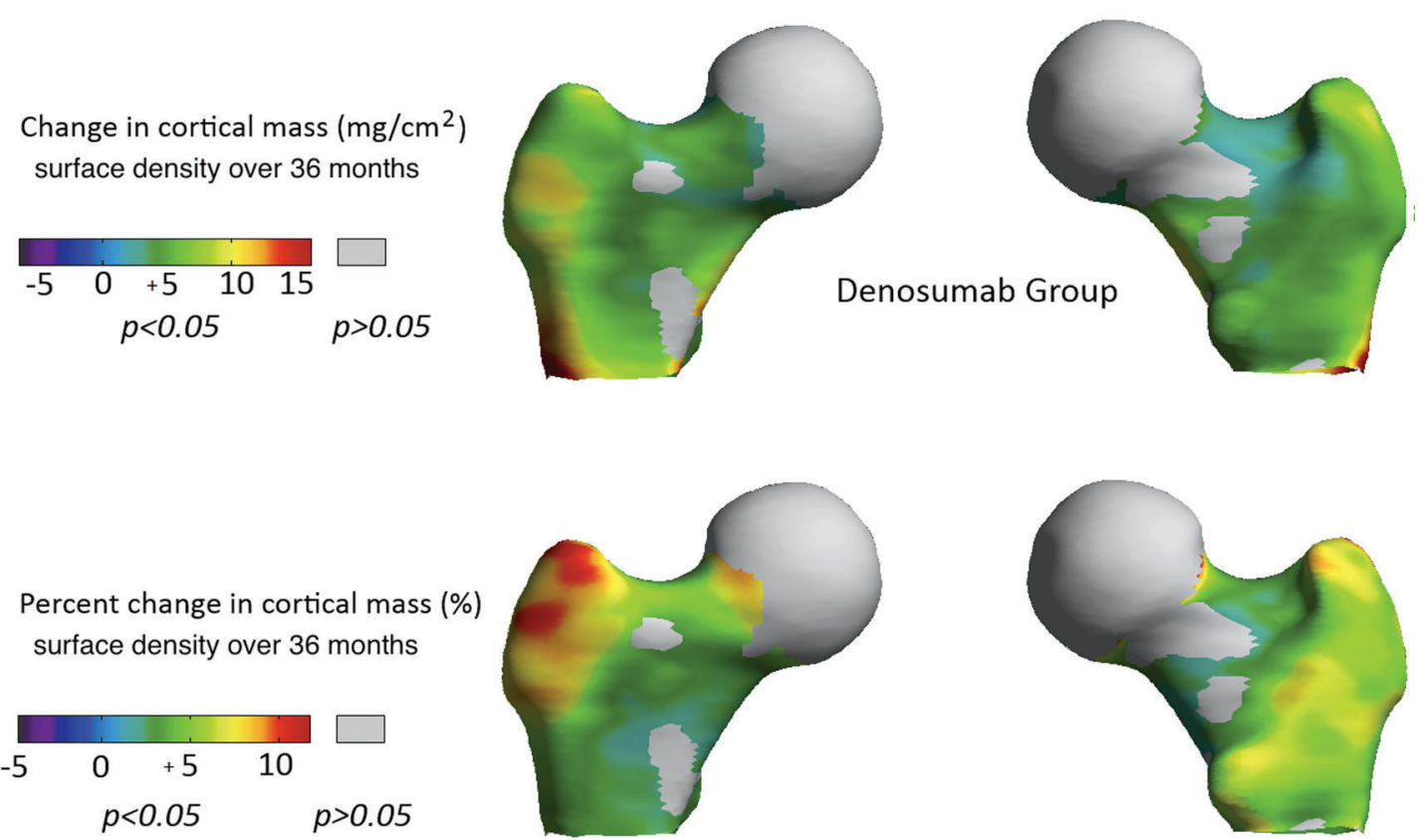

Fig. 4. Scaled color maps showing the changes in femoral cortical mass surface density observed in the placebo group (upper four panels) with the changes in cortical mass surface density in the denosumab group (lower four panels) after 36 months ( $n=61$ ). Change is displayed as either absolute increase at each surface point or percentage increase at each surface point.

We, therefore, need to be careful in our interpretation of such cortical thickness increases, and this is discussed in the context of the results in Discussion.

\section{Results}

The basic results of our analyses (Fig. 3, right panel) do not involve SPM, but simply show the overall group cortical density for drug and placebo arms plotted over time (see whole femur average cortical density in the Materials and Methods). In the same figure, we show for each study arm the overall average of all surface measurement points on all femurs for thickness (Fig. 3, center panel) and mass surface density (Fig. 3, left panel). Compared with baseline, there was a statistically significant increase in overall average cortical mass surface density $\left(+5.9 \mathrm{mg} / \mathrm{cm}^{2}\right.$, $p=3.0 \times 10^{-18}$, Fig. 3, left panel) and cortical thickness $(26 \mu \mathrm{m}$, 
Change in cortical thickness ( $\mathrm{mm}$ ) over 36 months

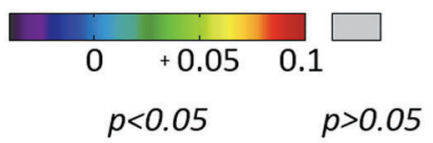

$p<0.05$ $p>0.05$

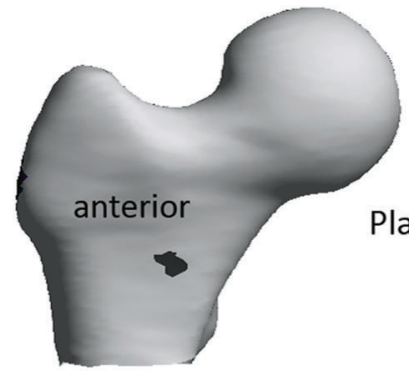

Placebo Group
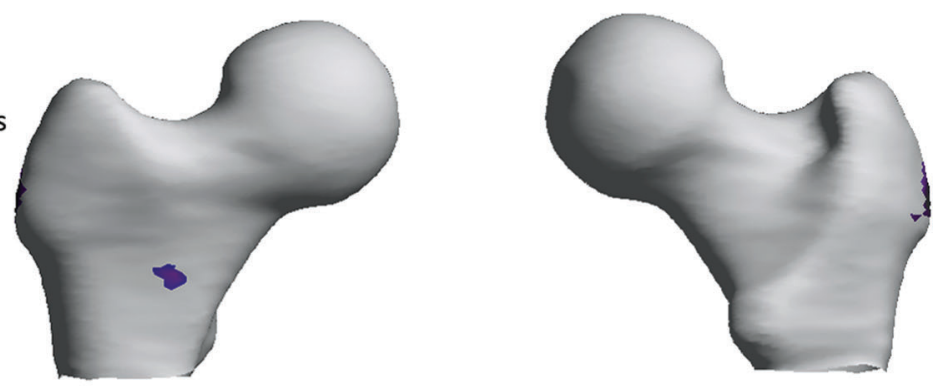

Percent change in cortical thicknes (\%) over 36 months

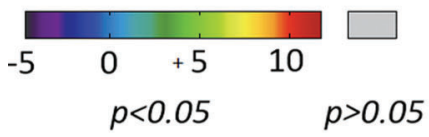

Change in cortical thickness $(\mathrm{mm})$ over 36 months

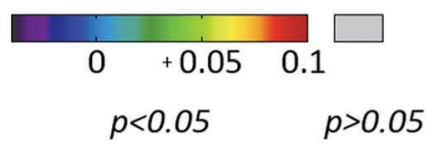

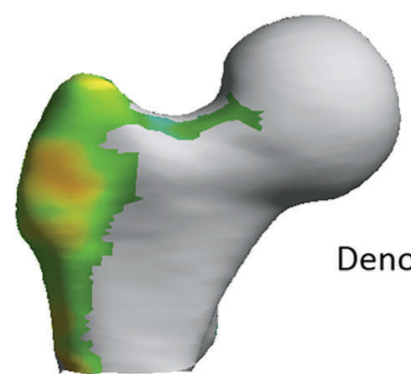

Denosumab Group

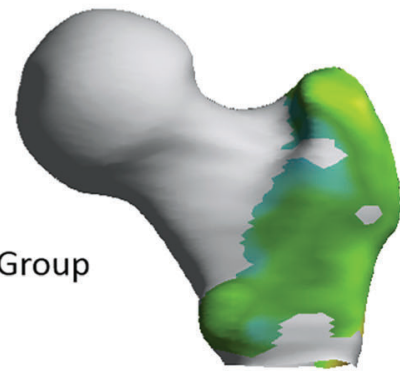

Percent change in cortical thickness (\%) over 36 months
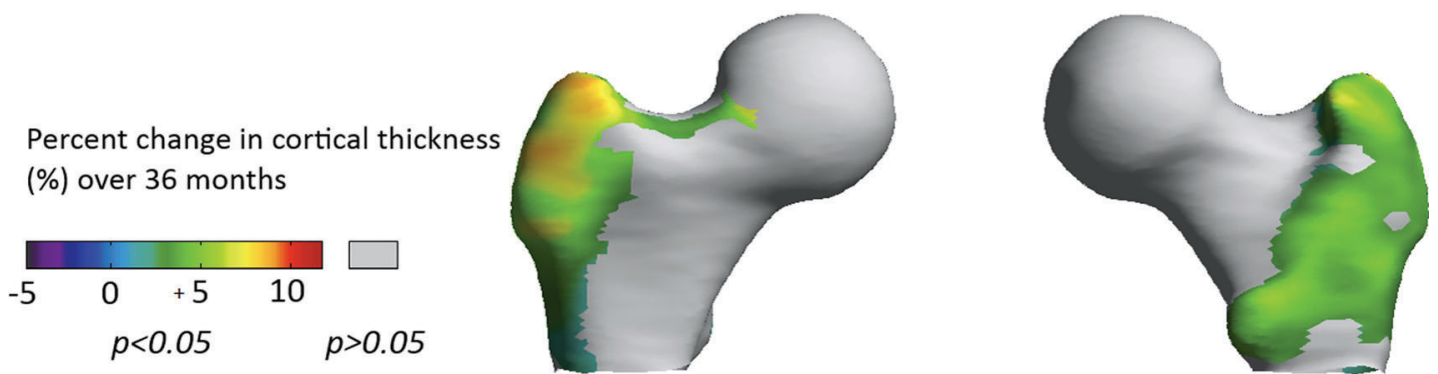

Fig. 5. Scaled color maps showing the changes in femoral cortical thickness observed in the placebo group (upper four panels) with the changes in cortical thickness for the denosumab group (lower four panels) after 36 months $(n=61)$. Thickness change is displayed as either absolute increase in cortical thickness $(\mathrm{mm})$ at each surface point or percentage increase at each surface point.

$p=3.2 \times 10^{-8}$, Fig. 2 center panel) in women treated with denosumab for 3 years (six injections). There was also a statistically significant increase in overall average cortical density $\left(+23 \mathrm{mg} / \mathrm{cm}^{3}, p=3.4 \times 10^{-12}\right.$, Fig. 3, right panel) in those receiving denosumab. There was a reduction in cortical mass surface density and thickness across much of the femoral surface in women who received placebo for 3 years, and in some focal areas, this also reached statistical significance by SPM (Fig. 4, upper panels). Cortical density increased transiently at 12 months in placebo-treated (calcium/vitamin D-receiving) patients (Fig. 3, right panel).

In Fig. 4, the color maps of the upper panels highlight the decrease in absolute and percentage cortical mass surface density observed with placebo at 36 months, whereas the lower panels show the increase observed with denosumab. In these results figures, only the statistically significant differences have 

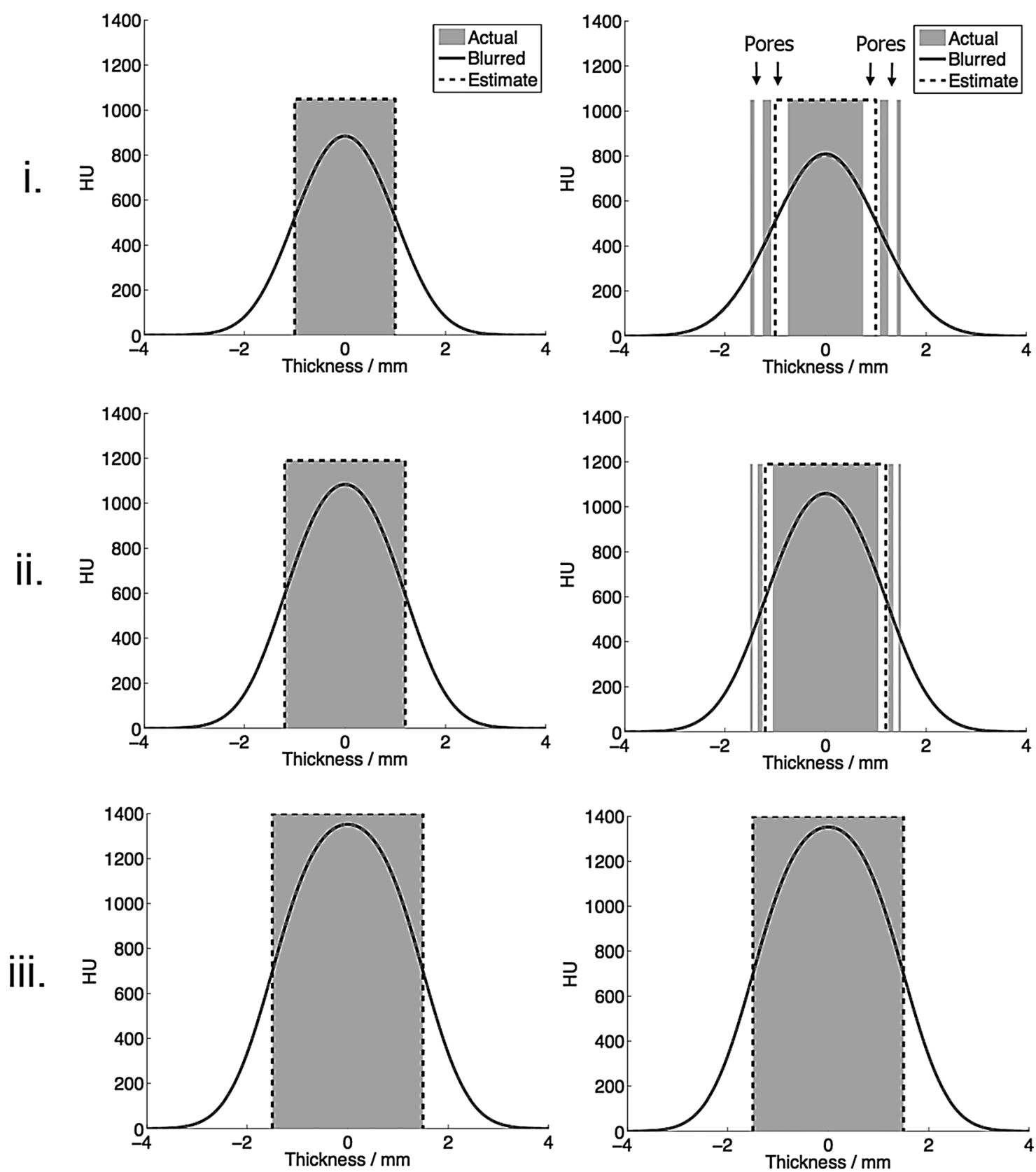

Fig. 6. Idealized representation of the effects of a drug on the measurement of thickness in a blurred CT cortex at one vertex (for an example of a single vertex, see red spot in Fig. 2). The drug progressively increases overall cortical density (HU) at three time points (i, ii, iii). Its other effect might be to add new bone on endosteal and periosteal surfaces (left panels) or alternatively to fill in extensive intracortical pores (right panels). With current cortical mapping techniques, both physiological processes would produce an identical increase in measured cortical thickness at that vertex.

been retained on the color map. Areas where there were no statistically significant differences are shown as a gray color. The same scales and anatomical perspectives (anterior and posterior view) of the average right femur are used in all the figures. By 3 years, most of the femoral cortex of women treated with denosumab displayed a statistically significant increase in cortical mass surface density relative to baseline (Fig. 4, lower panels). Intermediate time-point data (separate color maps for 12,24 , and 36 months) are shown in Supplemental Figs. S1 to S4. Color maps of the relative difference (denosumab relative to placebo group) are shown in Supplemental Figs. S5 and S6. The largest relative increase with denosumab was in the lateral greater trochanter (up to 12\%) close to the insertion site of the gluteal muscles. This site plus the inferior femoral neck and lateral shaft were also notable areas of absolute increase in cortical mass surface density.

In the lower panels of Fig. 5, the changes in cortical thickness for the denosumab group at 36 months are displayed. As mentioned in Materials and Methods, these results could reflect new bone or infilling of intracortical pores in existing bone. Overall, the extent of the increase in cortical mass surface density with denosumab was larger than that observed in cortical 
thickness. The largest relative increase in thickness was also found in the lateral greater trochanter (up to 12\%) at the insertion site of the gluteal muscles.

\section{Discussion}

We know that women with osteoporosis (who have generally thinner and more porous bones) are more likely to suffer hip fracture. However, rather than studying the overall hip bone density, it has become scientifically (and possibly clinically) more important to assess small focal areas of the femur because highquality simulations show that failure of just $4.5 \%$ of bone tissue in the proximal femur can be enough to cause catastrophic failure of the femoral neck. ${ }^{(25)}$ This catastrophic failure inevitably involves the outer cortical bone of the femur, ${ }^{(16)}$ which thins rapidly with age ${ }^{(17,26)}$ and is a key determinant of bone strength and fracture risk, ${ }^{(9,18,26-28)}$ although trabecular bone may be the first site to give way. Cortical bone thinning, in particular, is partially reversible, as we showed with previous work involving teriparatide therapy. ${ }^{(19,29)}$ Trabecular bone and subcortical trabecular bone are also key determinants of bone strength and fracture risk but have not been studied here. In this study, we tested the hypothesis that denosumab treatment of women with osteoporosis would improve cortical bone in regions of focal thinning and, thus, begin to explain the $8.4 \%$ increase in overall femur strength with treatment shown by modeling. We found that by 36 months, most of the femoral cortex of denosumab-treated women displayed a statistically significant increase in cortical mass surface density, but smaller foci were already detectable by 12 months. Specifically, the greatest relative increase in cortical mass surface density of up to $12 \%$ (Fig. 4) occurred in the lateral trochanter, a site where focal thinning has been linked with trochanteric fracture. ${ }^{(14)}$ Denosumab also increased cortical mass surface density in the femoral neck, a site that is important in the pathogenesis of hip fracture. ${ }^{(5,14)}$ Both femoral neck and trochanteric hip fractures were reduced by denosumab in the FREEDOM trial (in women aged 75 years and older, treatment halved the number of femoral neck fractures and reduced trochanteric fractures by 70\%). Because women treated with denosumab had fewer hip fractures, we speculate that the enhancement of cortical bone at key at-risk sites of the femur might have contributed to the efficacy of the drug.

Cortical bone mapping uses images from clinically acquired pelvic CT scans, such as those performed on participants in major osteoporosis trials. We previously discovered that the technique was effective in identifying focal thickening of femoral cortical bone during teriparatide treatment of women with severe osteoporosis. ${ }^{(19)}$ Because there was little change in cortical mass surface density in that study, ${ }^{(13)}$ it is likely that new but relatively undermineralized bone had been deposited or, alternatively, that new bone apposition from teriparatide administration was associated with increased intracortical porosity. ${ }^{(12,30)}$ In the present study, denosumab had a large effect on cortical mass surface density as well as thickness. Mass surface density can be increased by increasing the deposition and mineralization of bone or by infilling of pores. We have already highlighted the difficulty of distinguishing cortical thickness increases owing to increasing true bone thickness and the infilling of endocortical pores at a histological level. Fig. 6 shows how filling-in of intracortical pores can resemble increasing absolute thickness using cortical bone mapping, in a scenario where bone density is also increasing with time. Denosumab is considered a powerful anticatabolic, strongly inhibiting osteoclast formation, resorption activity, and enhancing bone mineralization through reduced bone remodeling. Cortical thickening is an intriguing and somewhat counterintuitive finding, given the expected antiresorptive profile of denosumab. Previous studies conducted in nonhuman experimental models ${ }^{(31)}$ or high-resolution scans of peripheral bones of human distal tibias or radii ${ }^{(32)}$ point to reduced cortical porosity as a therapeutic effect. Our results add to these by showing that denosumab has both general and focal effects on enhancing femoral cortical bone thickness (which might reflect infilling of cortical pores; Fig. 4).

A further limitation of the current analysis method is that we have deliberately focused exclusively on the cortical compartment. There is strong evidence that trabecular bone is critical in determining fracture resistance. ${ }^{(3)}$ Our bone mapping technique can now be applied to the trabecular compartment, and analyzing the effects of osteoporosis drugs on trabecular bone is a priority for future work. Finally, in this analysis, no allowance was made for patient age, height, or any other demographics at baseline, which might have influenced results. However, it is highly unlikely that failing to adjust for baseline values would have a systematic effect on the study results.

An increase in 3D bone density was also found by Genant and colleagues using the MIAF femur package (a QCT analysis tool allowing density estimation in various hip subregions). They found improvements of $5.4 \%$ to $9.9 \%$ in volumetric BMD after denosumab treatment for 3 years, with the highest-percentage improvement found in the trabecular compartment, but higher absolute changes in cortical density. ${ }^{(33)}$ Our analysis adds spatial information to these results, offering a plausible explanation for the robust reduction in hip fractures observed in FREEDOM.

In the present study, we discovered that femoral cortical bone in women with osteoporosis responds rapidly to denosumab treatment, particularly in the trochanteric region. This mechanism could enhance resistance to trauma, thereby explaining the rapid and persisting decrease in hip fractures found in denosumab-treated women with osteoporosis.

\section{Disclosures}

KESP and GMT are inventors on a related patent application GB0917524.1, "Image data processing systems." This does not alter the authors' adherence to all the JBMR policies on sharing data and materials. KESP is a consultant, speaker, and an advisory board member for Amgen Inc. and has received research grant support from Amgen Inc. GMT and AHG have received research grant support from Amgen Inc. MRM has received research grant support from Amgen Inc. and was an advisory board member for Amgen Inc. JPB is a consultant, speaker, and an advisory board member for Amgen Inc. and has received research grant support and honoraria from Amgen Inc. CL and AW are employees of and own stock or stock options in Amgen Inc.

\section{Acknowledgments}

The authors acknowledge all the investigators and study participants involved in the FREEDOM trial, as well as Synarc Imaging for coordinating and sharing the scan data. 
This study was funded by Amgen Inc., Thousand Oaks, CA, USA. Cambridge Bone Group is supported by Arthritis Research UK, The Evelyn Trust, and Cambridge NIHR Biomedical Research Centre.

Authors' roles: KESP, GMT, AHG, CL, AW, JPB, and MRM contributed to the conception and design of the study analysis. KESP, GMT, and AHG performed the analysis and interpretation of the data. All authors participated in drafting or revising the manuscript and approved the final version of the manuscript for submission. KESP, GMT, and AHG take responsibility for the integrity of the data analysis.

\section{References}

1. Cummings SR, San Martin J, McClung MR, et al. Denosumab for prevention of fractures in postmenopausal women with osteoporosis. N Engl J Med. 2009;361:756-65.

2. Parker MJ, Twemlow TR. Spontaneous hip fractures, $44 / 872$ in a prospective study. Acta Orthop Scand. 1997;68:325-6.

3. Nawathe $S$, Akhlaghpour $H$, Bouxsein ML, Keaveny TM. Microstructural failure mechanisms in the human proximal femur for sideways fall loading. J Bone Miner Res. 2014;29:507-15.

4. Keaveny $T$, McClung $M$, Genant $H$, et al. Femoral and vertebral strength improvements in postmenopausal women with osteoporosis treated with denosumab. J Bone Miner Res. 2014;29:158-65.

5. Cristofolini L, Juszczyk M, Martelli S, Taddei F, Viceconti M. In vitro replication of spontaneous fractures of the proximal human femur. J Biomech. 2007;40:2837-45.

6. Cristofolini L, Juszczyk M, Taddei F, Viceconti M. Strain distribution in the proximal human femoral metaphysis. Proc Inst Mech Eng $\mathrm{H}$. 2009;223:273-88.

7. Viceconti M, Taddei F, Cristofolini L, Martelli S, Falcinelli C, Schileo E. Are spontaneous fractures possible? An example of clinical application for personalised, multiscale neuro-musculo-skeletal modelling. J Biomech. 2012;45:421-6.

8. Zimmermann EA, Schaible E, Bale $\mathrm{H}$, et al. Age-related changes in the plasticity, toughness of human cortical bone at multiple length scales. Proc Natl Acad Sci USA. 2011;108:14416-21.

9. Carballido-Gamio J, Harnish R, Saeed I, et al. Proximal femoral density distribution and structure in relation to age and hip fracture risk in women. J Bone Miner Res. 2013;28:537-46.

10. Thomas CD, Mayhew PM, Power J, et al. Femoral neck trabecular bone: loss with aging and role in preventing fracture. J Bone Miner Res. 2009;24:1808-18.

11. Black DM, Bouxsein ML, Marshall LM, et al. Proximal femoral structure and the prediction of hip fracture in men: a large prospective study using QCT. J Bone Miner Res. 2008;23:1326-33.

12. Treece GM, Gee AH, Mayhew PM, Poole KE. High resolution cortical bone thickness measurement from clinical CT data. Med Image Anal. 2010;14:276-90.

13. Treece GM, Poole KE, Gee AH. Imaging the femoral cortex: thickness, density and mass from clinical CT. Med Image Anal. 2012;16:952-65.

14. Poole KE, Treece GM, Mayhew PM, et al. Cortical thickness mapping to identify focal osteoporosis in patients with hip fracture. PloS One. 2012;7:e38466.

15. Bessho M, Ohnishi I, Matsumoto T, et al. Prediction of proximal femur strength using a CT-based nonlinear finite element method: differences in predicted fracture load and site with changing load and boundary conditions. Bone. 2009;45:226-31.

16. de Bakker PM, Manske SL, Ebacher V, Oxland TR, Cripton PA, Guy P. During sideways falls proximal femur fractures initiate in the superolateral cortex: evidence from high-speed video of simulated fractures. J Biomech. 2009;42:1917-25.
17. Poole KE, Mayhew PM, Rose CM, et al. Changing structure of the femoral neck across the adult female lifespan. J Bone Miner Res. 2010;25:482-91.

18. Johannesdottir F, Poole KE, Reeve J, et al. Distribution of cortical bone in the femoral neck and hip fracture: a prospective case-control analysis of 143 incident hip fractures; the AGES-REYKJAVIK Study. Bone. 2011;48:1268-76.

19. Poole KE, Treece GM, Ridgway GR, Mayhew PM, Borggrefe J, Gee AH. Targeted regeneration of bone in the osteoporotic human femur. PloS One. 2011;6:e16190.

20. McClung MR, Zanchetta JR, Hoiseth A, et al. Denosumab densitometric changes assessed by quantitative computed tomography at the spine and hip in postmenopausal women with osteoporosis. J Clin Densitom. 2013;16:250-6.

21. Rueckert $D$, Frangi $A F$, Schnabel JA. Automatic construction of 3-D statistical deformation models of the brain using nonrigid registration. IEEE Trans Med Imaging. 2003;22:1014-25.

22. Friston KJ, Holmes AP, Worsley KJ, Poline J-P, Frith CD, Frackowiak RSJ. Statistical parametric maps in functional imaging: a general linear approach. Hum Brain Mapping. 1994;2:189-210.

23. Worsley K, Taylor J, Carbonell F, et al. SurfStat: a Matlab toolbox for the statistical analysis of univariate and multivariate surface and volumetric data using linear mixed effects models and random field theory. Presented at: Neurolmage Organization for Human Brain Mapping 2009 Annual Meeting; San Francisco, CA. Neurolmage. 2009;47:S102.

24. Genant HK, Keaveney T, Zapalowski C, et al. Cortical bone parameters at the hip in response to denosumab vs placebo and the clinical relevance of these changes in postmenopausal women with osteoporosis $<75$ and $\geq 75$ years old. J Bone and Miner Res. 2013;28Suppl 1:S180.

25. Wainwright $S A$, Marshall LM, Ensrud $K E$, et al. Hip fracture in women without osteoporosis. J Clin Endocrinol Metab. 2005;90: 2787-93.

26. Mayhew PM, Thomas CD, Clement JG, et al. Relation between age, femoral neck cortical stability, and hip fracture risk. Lancet. 2005;366:129-35.

27. Gluer CC, Cummings SR, Pressman A, et al. Prediction of hip fractures from pelvic radiographs: the study of osteoporotic fractures. The Study of Osteoporotic Fractures Research Group. J Bone Miner Res. 1994;9:671-7.

28. Holzer G, von Skrbensky G, Holzer LA, Pichl W. Hip fractures and the contribution of cortical versus trabecular bone to femoral neck strength. J Bone Miner Res. 2009;24:468-74.

29. Borggrefe J, Graeff C, Nickelsen TN, Marin F, Gluer CC. Quantitative computed tomography assessment of the effects of 24 months of teriparatide treatment on 3-D femoral neck bone distribution, geometry and bone strength: results from the EUROFORS study. J Bone Miner Res. 2009;25:472-81.

30. Sato $M$, Westmore $M, M a Y L$, et al. Teriparatide [PTH(1-34)] strengthens the proximal femur of ovariectomized nonhuman primates despite increasing porosity. J Bone Miner Res. 2004; 19:623-9.

31. Kostenuik PJ, Smith SY, Jolette J, Schroeder J, Pyrah I, Ominsky MS. Decreased bone remodeling and porosity are associated with improved bone strength in ovariectomized cynomolgus monkeys treated with denosumab, a fully human RANKL antibody. Bone. 2011;49:151-61.

32. Seeman E, Delmas PD, Hanley DA, et al. Microarchitectural deterioration of cortical and trabecular bone: differing effects of denosumab and alendronate. J Bone Miner Res. 2010;25: 1886-94.

33. Genant HK, Libanati C, Engelke K, et al. Improvements in hip trabecular, subcortical, and cortical density and mass in postmenopausal women with osteoporosis treated with denosumab. Bone. 2013:56:482-8. 